\title{
Switch to Combined GLP1 Receptor Agonist Lixisenatide with Basal Insulin Glargine in Poorly Controlled T2DM Patients with Premixed Insulin Therapy: A Clinical Observation and Pilot Study in Nine Patients
}

Jürgen Harreiter · Lana Kosi-Trebotic · Albert Lukas · Peter Wolf • Yvonne Winhofer •

Anton Luger · Alexandra Kautzky-Willer · Michael R. Krebs (D)

Received: February 6, 2017 / Published online: March 29, 2017

(c) The Author(s) 2017. This article is an open access publication

\section{ABSTRACT}

Introduction: To prove the feasibility and safety of a conversion to once-daily injected GLP1 agonist (lixisenatide) and long-acting basal insulin analogue (glargine) in patients with T2DM and poorly controlled glycemia previously treated with multiple injections of premixed insulins (iPremix) in an outpatient setting.

Methods: Nine patients with T2DM currently receiving iPremix formulations and poor glycemic control were switched to once-daily injected lixisenatide (Lixi) and basal insulin analogue glargine (iGlar) for a 12-week period. Efficacy was defined as A1c reduction of at least $0.4 \%$ and weight loss of $0.5 \mathrm{~kg}$ or higher.

Results: Five of nine patients achieved A1c reductions of $0.4 \%(4 \mathrm{mmol} / \mathrm{mol})$ or higher and six of nine patients a weight loss of $0.5 \mathrm{~kg}$ or higher. A mean $\mathrm{A} 1 \mathrm{C}$ reduction of $0.5 \% \pm 0.5 \%$ $(6 \mathrm{mmol} / \mathrm{mol})$ and mean weight loss of $-1.4 \pm 3.6 \mathrm{~kg}$ were observed in all patients. Total

Enhanced Content To view enhanced content for this article go to http://www.medengine.com/Redeem/46F7 F0602FD50329.

J. Harreiter · L. Kosi-Trebotic · A. Lukas · P. Wolf .

Y. Winhofer · A. Luger · A. Kautzky-Willer ·

M. R. Krebs $(\bowtie)$

Division of Endocrinology and Metabolism,

Department of Medicine III, Medical University of

Vienna, Währingergürtel 18-20, 1090 Vienna,

Austria

e-mail: michael.krebs@meduniwien.ac.at daily insulin dose after 12 weeks declined from $56 \pm 26 \mathrm{IU}$ with iPremix formulations to $47 \pm 17$ IU in patients taking combined iGlar and Lixi. Corrections with fast acting insulin glulisine (iGlu) were necessary in two patients on a regular basis and in four patients on an irregular basis (2.3 IU mean total daily dose). Two patients did not need additional iGlu. Postprandial glucose profiles were lower in the combined group compared with iPremix throughout the day, which resolved in the afternoon. No metabolic derangements occurred. Mild hypoglycemia and gastrointestinal symptoms were the most often reported adverse events affecting three patients. Conclusion: The conversion to once-daily injected GLP1 agonist Lixi and basal iGlar could safely be performed in an outpatient setting and was associated with better postprandial glycemic control throughout the day, except dinner, compared to iPremix.

Clinical Trial Registration: EU clinical trials register EudraCT number 2013-005334-37 and ClinicalTrials.gov NCT02168491.

Funding: Sponsored by the Medical University of Vienna and in part supported by Sanofi-Aventis.

Keywords: Diabetes management; Diabetes therapy; GLP1 receptor agonist; Insulin glargine; Lixisenatide; Long acting insulin; Premixed insulin; Type 2 diabetes mellitus 


\section{INTRODUCTION}

The prevalence of type 2 diabetes mellitus (T2DM) is increasing worldwide with a doubling of numbers expected within the next three decades [1]. To overcome progressive decline of insulin secretion over the years of disease progression a subsequent dependency on subcutaneous insulin administration follows oral glucose-lowering medication [2]. Obviously flexible, intensified insulin therapy regimens would be the best option for these patients to cover postprandial insulin need. However, clinical inertia among physicians, patient difficulties to manage T2DM with complex therapeutic schemes, and/or lack of patient adherence and health disparities might hamper optimal glucose management [3]. In Austria and several other countries around the world premixed insulin (iPremix) regimens are used routinely for treatment of those patients in need of a simplified approach beyond basal insulin, as the efficacy is widely proven, fewer injections are necessary, and beneficial postprandial glucose levels compared with basal insulin treatment are reported [2, 4]. However, a well-known higher risk of hypoglycemia exists $[2,4]$.

Recently new coformulations with combinations of GLP1 receptor agonists (GLP1-RA) and long-acting insulin analogues were launched and shown to be effective and safe in treatment of T2DM [5-10]. However, it is unknown if patients with T2DM previously treated with iPremix with poorly controlled glycemia are able to be treated effectively by a new combined coformulation administered once daily, potentially facilitating insulin injection and possibly increasing adherence.

We hypothesized that a switch from iPremix to a combination therapy of GLP1-RA lixisenatide (Lixi; Lyxumia ${ }^{\circledR}$, Sanofi, Paris, France) and basal insulin glargine (iGlar; Lantus ${ }^{\circledR}$, Sanofi, Paris, France) in patients with T2DM is practicable in an outpatient setting. The aim of this study was to prove the principle of feasibility, effectiveness, and safety of once-daily injected GLP1-RA lixisenatide (Lixi) and basal insulin glargine (iGlar) combined in patients with
T2DM and poor glycemic control previously treated with at least twice-daily iPremix.

\section{METHODS}

This study is an exploratory proof of principle, monocentric, non-randomized clinical observation study including 11 patients with poorly controlled T2DM with iPremix. Patients with T2DM were recruited from the diabetes outpatient ward of the Department of Endocrinology and Metabolism at the Medical University of Vienna/General Hospital Vienna, Austria. Glucose metabolism was observed before treatment start and compared with profiles after combined treatment. All procedures followed were in accordance with the ethical standards of the responsible committee on human experimentation (institutional and national) and with the Helsinki Declaration of 1964, as revised in 2013. Informed consent was obtained from all patients for being included in the study.

Main inclusion criteria included type 2 diabetes, age greater than 18 years and less than 75 years, A1c $7 \%$ to $11 \%(53-97 \mathrm{mmol} / \mathrm{mol})$, and written informed consent. Exclusion criteria included women of reproductive age, loss of hypoglycemia sensibility, oral glucose-lowering medication except metformin, chronic liver or kidney disease, dyslipidemia, history of pancreatitis or pancreatic tumors, and history of psychiatric disease or autoimmune disease. Recruitment started in December 2014 and lasted till July 2015.

After screening, patients were treated with iPremix for another week. In this period home glucose self-monitoring with at least seven daily measurements was performed (fasting, before main courses, $2 \mathrm{~h}$ after meals, before sleeping). After 1 week, iPremix was changed to iGlar with a dose reduction to $60 \%$ of the last total daily iPremix dose. At the same time Lixi was started with a dose of $10 \mu \mathrm{g} /$ day with subsequent up-titration to $20 \mu \mathrm{g} /$ day after 2 weeks, if well tolerated. iGlar dose was regularly up-titrated at every study visit according to glucose profiles. Total treatment duration was 12 weeks. To prevent hyperglycemic events patients were 
trained to inject fast-acting insulin glulisine (iGlu, Apidra ${ }^{\circledR}$, Sanofi, Paris, France) if glucose levels exceed $200 \mathrm{mg} / \mathrm{dl}$. Training was individualized and correction rates were adapted to patients' needs as follows: $200-250 \mathrm{mg} / \mathrm{dl}$, 2 units; $250-300 \mathrm{mg} / \mathrm{dl}, 4$ units; and greater than $300 \mathrm{mg} / \mathrm{dl}, 6-8$ units of fast-acting insulin were injected by the patient. If glucose levels exceed $400 \mathrm{mg} / \mathrm{dl}$ or glucose levels constantly exceed $300 \mathrm{mg} / \mathrm{dl}$, patients were instructed to contact the study site immediately for further instruction or hospitalization if necessary. At every visit urinary testing for ketone bodies was performed. To ensure safety in the first week after switching from iPremix to combined Lixi and iGlar, regular visits or telephone calls were performed.

At screening, medical history and current medication were obtained by questioning or from medical records. The patient was examined by a trained physician to rule out any condition excluding the patient from the trial. Vital signs and a 12-lead ECG were performed. Last menstruation time was asked in women to examine reproductive function. Blood samples were analyzed in our ISO9001 certified central laboratory. All methods including reference parameters are available from www.kimcl.at. The use of glucometers was retrained for home blood glucose self-monitoring, as well as maintenance of glucose profiles with several distinct measure points.

Descriptive data analysis was performed for all parameters. Continuous variables were summarized by mean \pm SD as well as median and 25th and 75th percentiles and categorical variables by counts and percentages. Clinical efficacy was defined as A1c reduction of at least $0.4 \%(4 \mathrm{mmol} / \mathrm{mol})$ and weight loss of at least $0.5 \mathrm{~kg}$. Continuous variables were analyzed using Wilcoxon signed-rank test. Given the small sample size a non-parametric test was used for paired samples. Areas under the curves were calculated using trapezoidal rule.

Statistical analysis was performed using SPSS 24.0 (SPSS Inc, Chicago, IL, USA) and GraphPad Prism 5 (GraphPad Software Inc, La Jolla, CA, USA). A two-sided $p$ value less than 0.05 was considered statistically significant.

\section{RESULTS}

In total 11 patients with T2DM were screened, two of whom discontinued immediately after screening because of time limitations; thus nine patients were switched to combined Lixi and iGlar treatment of which two were female (Table 1). Another patient finished prematurely because of skepticism against new treatment procedures, although an obvious amelioration of glycemic control with A1c reduction from $10.7 \%(93 \mathrm{mmol} / \mathrm{mol})$ to $9.6 \%(81 \mathrm{mmol} / \mathrm{mol})$ and no side effects were reported.

Twelve weeks combined Lixi and iGlar treatment was associated with a reduction in $\mathrm{A} 1 \mathrm{c}$ of at least $0.4 \%$ (min. -0.4 , max. $-1.4 \%$; $4 \mathrm{mmol} / \mathrm{mol}$, min. -4 , max. $-15 \mathrm{mmol} / \mathrm{mol}$ ) in 5 of $9(56 \%)$ patients. In these responders a mean reduction of $\mathrm{A} 1 \mathrm{C}$ of $0.9 \pm 0.4 \%$ $(10 \pm 4 \mathrm{mmol} / \mathrm{mol})$ was seen, whereas 3 of 9 (33\%) non-responders had a reduction of only $0.1 \%$ and one patient had a $0.1 \%$ A1C increase ( $1 \mathrm{mmol} / \mathrm{mol}$ both). A significant total A1c reduction of $0.5 \pm 0.5 \%(6 \pm 6 \mathrm{mmol} / \mathrm{mol})$ was seen in all patients $[Z=-2.44,95 \%$ CI 0.1 , $1.0 \%(1,10 \mathrm{mmol} / \mathrm{mol}) ; p<0.02]$. Only 1 of 9 (11\%) patients achieved A1c less than $7.0 \%$ $(53 \mathrm{mmol} / \mathrm{mol})$. A clear tendency of better postprandial glycemic control was visible after 12 weeks of treatment with combined Lixi and iGlar compared to iPremix (Fig. 1). This effect was especially pronounced during the day and was suspended in the evening. However, AUCs were not significantly different (AUC iPremix mean \pm Std $2927.3 \pm 772.9 \mathrm{mg} / \mathrm{dl} \times \mathrm{min}$, median $3155.0 \mathrm{mg} / \mathrm{dl} \times \mathrm{min}, 25-75$ th percentile 2257.8-3469.3 mg/dl $\times$ min; AUC Lixi + iGlar $2697 \pm 807.6, \quad 2781.0, \quad 2207.0-3082.2 \mathrm{mg} /$ $\mathrm{dl} \times \min ; Z=-1.36 p=0.17)$.

In 6 of $9(67 \%)$ participants a weight loss of at least $0.5 \mathrm{~kg}$ (min. $-0.6 \mathrm{~kg}$, max. $-7.8 \mathrm{~kg}$ ) was observed, two patients gained weight (2.8 and $4.1 \mathrm{~kg}$ ), and one patient had no weight change at all. In responders a mean weight loss of $-3.2 \pm 2.5 \mathrm{~kg}$ and in all patients mean weight loss of $-1.4 \pm 3.6 \mathrm{~kg}$ were observed $(Z=-0.98$, $95 \%$ CI $-1.4,4.1 ; p=0.33$ ).

Combined Lixi and iGlar was associated with lower total daily insulin doses (47 $\pm 17 \mathrm{IU})$ after 
Table 1 Baseline characteristics of study participants with T2DM

\begin{tabular}{lllllc}
\hline & $\boldsymbol{N}$ & Mean & Std & Median & 25th-75th percentile \\
\hline Age (years) & 9 & 65.0 & 6.1 & 66.0 & $60.5-70.5$ \\
Height $(\mathrm{cm})$ & 9 & 171.0 & 5.6 & 174.0 & $164.5-175.0$ \\
Weight $(\mathrm{kg})$ & 9 & 94.9 & 10.4 & 97.9 & $88.1-101.5$ \\
BMI $\left(\mathrm{kg} / \mathrm{m}^{2}\right)$ & 9 & 31.0 & 3.4 & 32.0 & $28.8-33.1$ \\
BP sys $(\mathrm{mmHg})$ & 9 & 149 & 21 & 149 & $138-166$ \\
BP dia $(\mathrm{mmHg})$ & 9 & 79 & 11 & 78 & $72-85$ \\
HbAlc $(\%)$ & 9 & 9.1 & 1.1 & 9.0 & $8.7-9.9$ \\
HbAlc (mmol/mol) & 9 & 76 & 12 & 75 & $72-85$ \\
Heart rate (bpm) & 9 & 84 & 7 & 86 & $81-89$ \\
Waist (cm) & 9 & 112.0 & 5.7 & 112.0 & $107.5-117.5$ \\
Total daily insulin dose $(\mathrm{U})$ & 9 & 56.2 & 26.0 & 52.0 & $35.0-77.0$ \\
Diabetes duration (years) & 9 & 12.1 & 5.2 & 10.0 & $9.0-16.0$ \\
& & Counts & $\%$ & & \\
Caucasian ethnicity & $9 / 9$ & 100 & & \\
Male sex & & $7 / 9$ & 78 & & \\
Postmenopausal female & & $2 / 2$ & $100 \%$ & & \\
\hline
\end{tabular}

$T 2 D M$ type 2 diabetes mellitus, $N$ number, $S t d$ standard deviation, $B M I$ body mass index, $B P$ sys systolic blood pressure, $B P$ dia diastolic blood pressure

${ }^{a}$ Age of women in postmenopause (minimum-maximum), 57-70 years

12 weeks treatment compared to iPremix alone (56 \pm 26 IU) at baseline, which was not significantly different $(Z=-1.72,95 \%$ CI $-2,20$; $p=0.09$ ).

As per study protocol and as a safety measure we decided to reduce the basal insulin dose to $60 \%$ of the previously used iPremix. Especially at the beginning nearly all patients had to correct with fast-acting insulin. After 12 weeks of combined treatment additional iGlu corrections were necessary on a regular $(n=2)$ or irregular daily basis $(n=4)$. Two patients had no additional iGlu need (Table 2). Total daily insulin doses of iPremix, iGlar, and iGlu per patient at baseline and after 12 weeks of combined treatment are shown in Table 2. In total a mean daily iGlu dose of $2.3 \mathrm{IU}$ was administered, and 92 self blood glucose measurements of 404 measurements were above $200 \mathrm{mg} / \mathrm{dl} \quad(22.8 \%)$
Fig. 1 a Means and standard deviations at each time point of daily home blood glucose self-monitoring at baseline (= iPremix, black) and after 12 weeks of combined treatment ( $=$ iGlarLix, gray) with combined lixisenatide and insulin glargine (no significant difference for AUC or fasting/postprandial glucose levels). b Insulin doses throughout the study. c Pre and post graphs showing changes of HbAlc. d Pre and post graphs showing changes of weight. e Pre and post graphs showing changes of waist circumference

compared to 141 elevated values of 305 measurements at baseline (46.3\%). The initial insulin reduction was too high and we constantly up-titrated iGlar doses to achieve acceptable fasting glucose levels (Fig. 1), which was unsuccessful.

During the 12 weeks one serious adverse event in one female patient who underwent an 
(a)

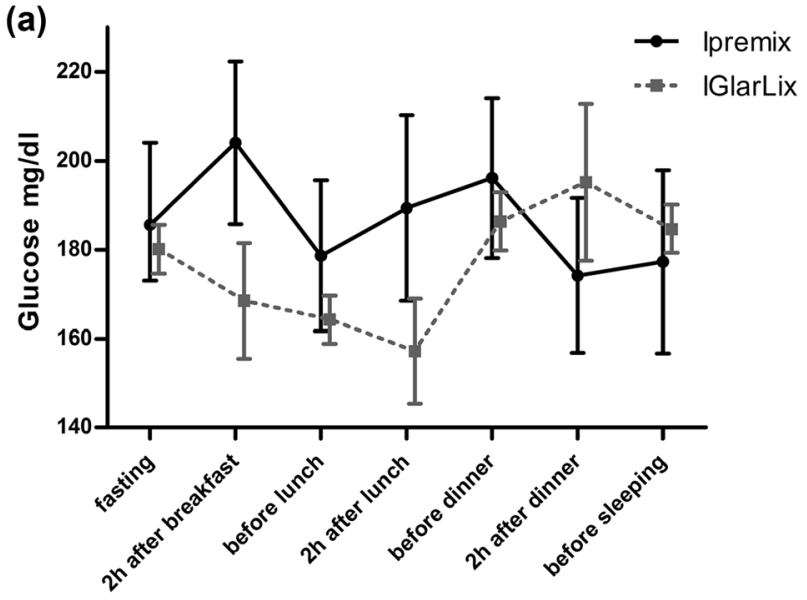

(c)

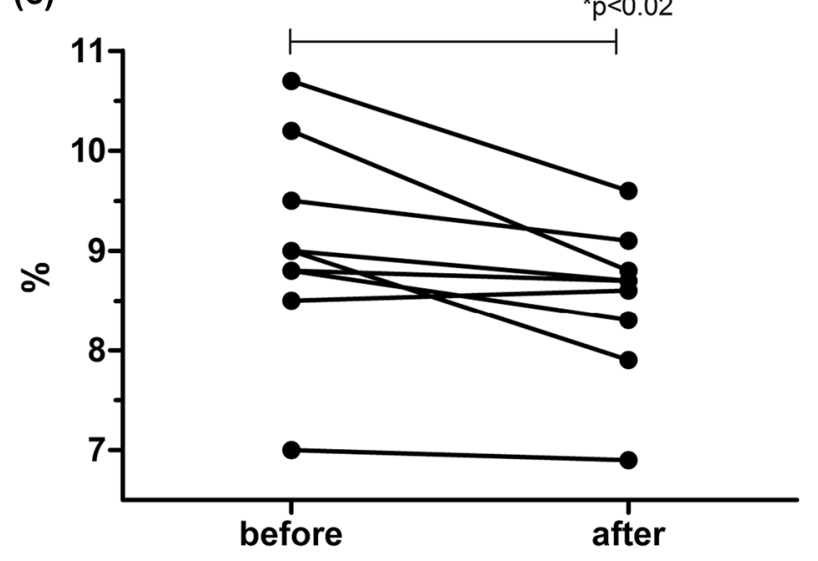

(b)

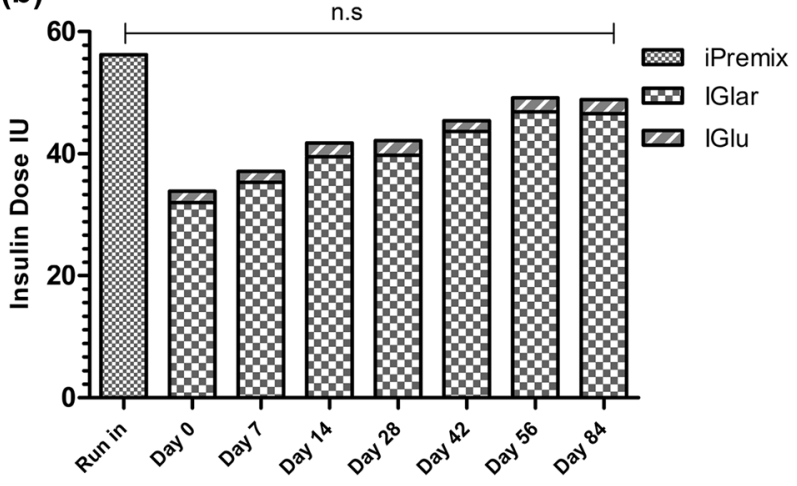

(d)

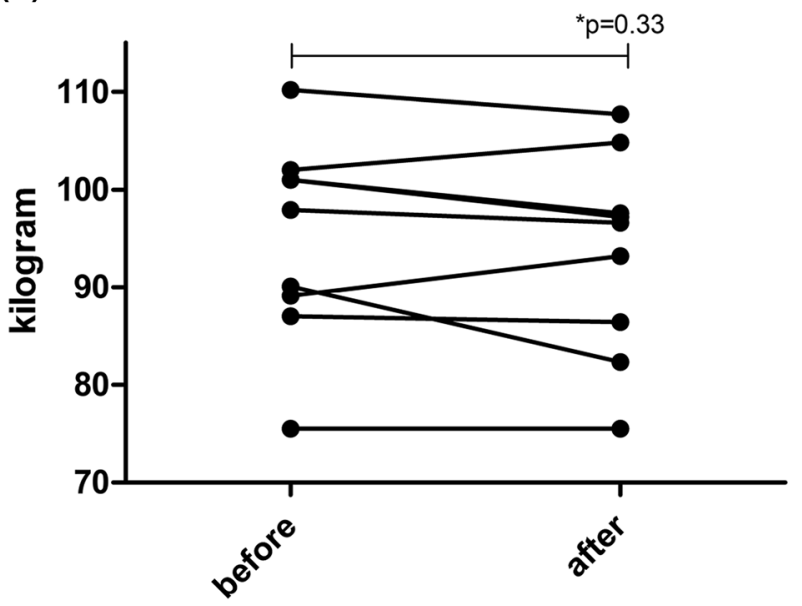

Waist

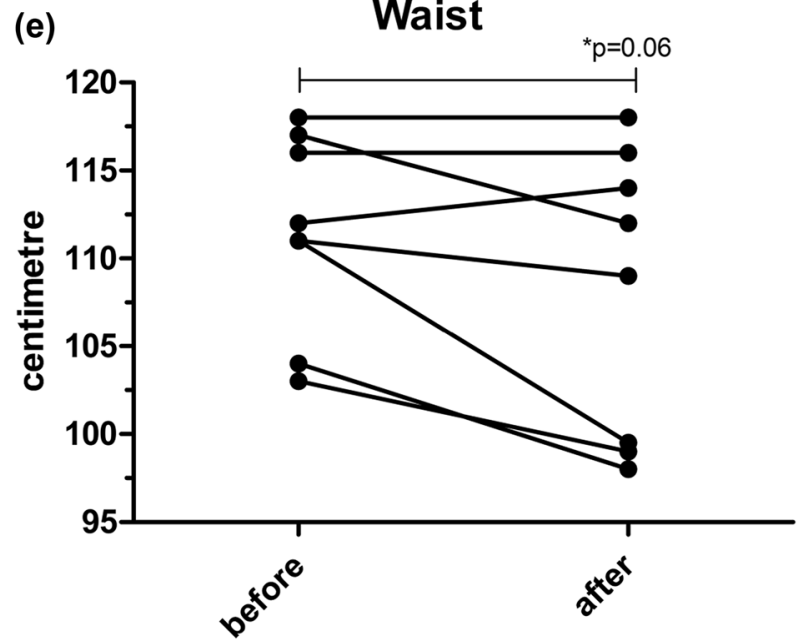


elective ENT surgery with no postsurgical complications was reported. Several adverse events have been reported, of which four were considered moderate and 18 of mild appearance. Most often $(n=8)$ mild asymptomatic hypoglycemia was reported and one symptomatic hypoglycemia was reported by three patients (33\%). Gastrointestinal complications were reported in two patients $(22 \%)$. Urinary tract infections occurred in two patients $(22 \%)$.

\section{DISCUSSION}

In this exploratory study we demonstrated that poorly controlled patients with long-standing T2DM and at least twice-daily iPremix can be safely and effectively treated with once-daily Lixi and iGlar. A majority of patients achieved clinically significant A1c level and weight reductions. A tendency towards an amelioration of postprandial glucose profiles during the day, except dinner, was seen using comparable insulin doses. Adverse events reported in a few patients were mostly mild hypoglycemia and mild gastrointestinal complaints.

Latest guidelines from the American Diabetes Association recommend to consider a combination of injectable therapies in patients insufficiently treated with combined oral drugs and basal insulin [11]. In these subjects one option is to start with a combination of GLP1-RA and basal insulin; other options include adding fast-acting insulin before the largest meal or changing to twice-daily premixed insulin injections. Further therapeutical escalation is needed in those patients not reaching A1c target goals or patients intolerant to this combination. However, so far there are no recommendations regarding patients not achieving treatment goals and/or suffering from hypoglycemia during treatment with multiple daily injections of premixed insulin. This highlights the clinical need for approaches that allow changing the therapeutic strategy in subjects with usually long-standing diabetes treated with high insulin doses. With our proof of principle study we could demonstrate that patients with established premixed insulin therapy injected at least twice daily can be safely switched to a combination therapy of GLP1-RA lixisenatide and basal insulin glargine administered once daily.

This small study corroborates recent findings of randomized controlled trials (RCTs) investigating the effects and safety of various combinations of once-daily Lixi with either iGlar compared to iGlar alone on top of metformin, or basal insulin compared to metformin and basal insulin, oral agents, or compared to placebo alone $[5,7,9,10,12]$. A further study-the LixiLan $\mathrm{O}$ trial-tested combined Lixi and iGlar and iGlar or Lixi alone [8]. In these studies better glycemic and postprandial control, significant lower A1c levels and weight loss were shown for combined iGlarLixi. Study findings of the LixiLan or GetGoal trials report comparable rates of hypoglycemic events (25-40\% vs. 33\%) and gastrointestinal adverse events (15-40\% vs. $22 \%$ ) in the combined iGlar and Lixi groups as found in our study [5, 7-10]. After initiation of Lixi with basal insulin a significant dose reduction of nearly 6 units was reported in the GetGoal L trial, which is of similar magnitude to that reported in our study [5].

One of the advantages of combined Lixi and iGlar treatment was a sufficient decrease of postprandial glucose levels after breakfast and lunch, but no suppression of glucose levels after dinner was noticed. This might be caused by the half-life of $2-4 \mathrm{~h}$ of the short-acting GLP-RA lixisenatide and displays a potential weakness of once-daily injected iGlar and Lixi combinations. Conversely, bigger studies including the LixiLan $\mathrm{O}$ trial demonstrated sufficient glucose level reductions throughout the day and also around dinner or thereafter after 30 weeks, albeit increasing glucose levels observed in daily profiles throughout the day [8].

In our study, the prevalence of responders with A1c reductions of at least $0.4 \%$ ( $4 \mathrm{mmol} / \mathrm{mol}$, 5 of 9 patients, 56\%) was low. In $44 \%$ of patients combined therapy did not reduce A1c sufficiently. In an RCT a glucose deterioration was found in $38 \%$ of patients with T2DM switched from insulin to exenatide, whereas in those who continued insulin glycemic control deteriorated in 19\% [13]. Worse glycemic control was associated with higher baseline insulin need, longer duration of T2DM, 


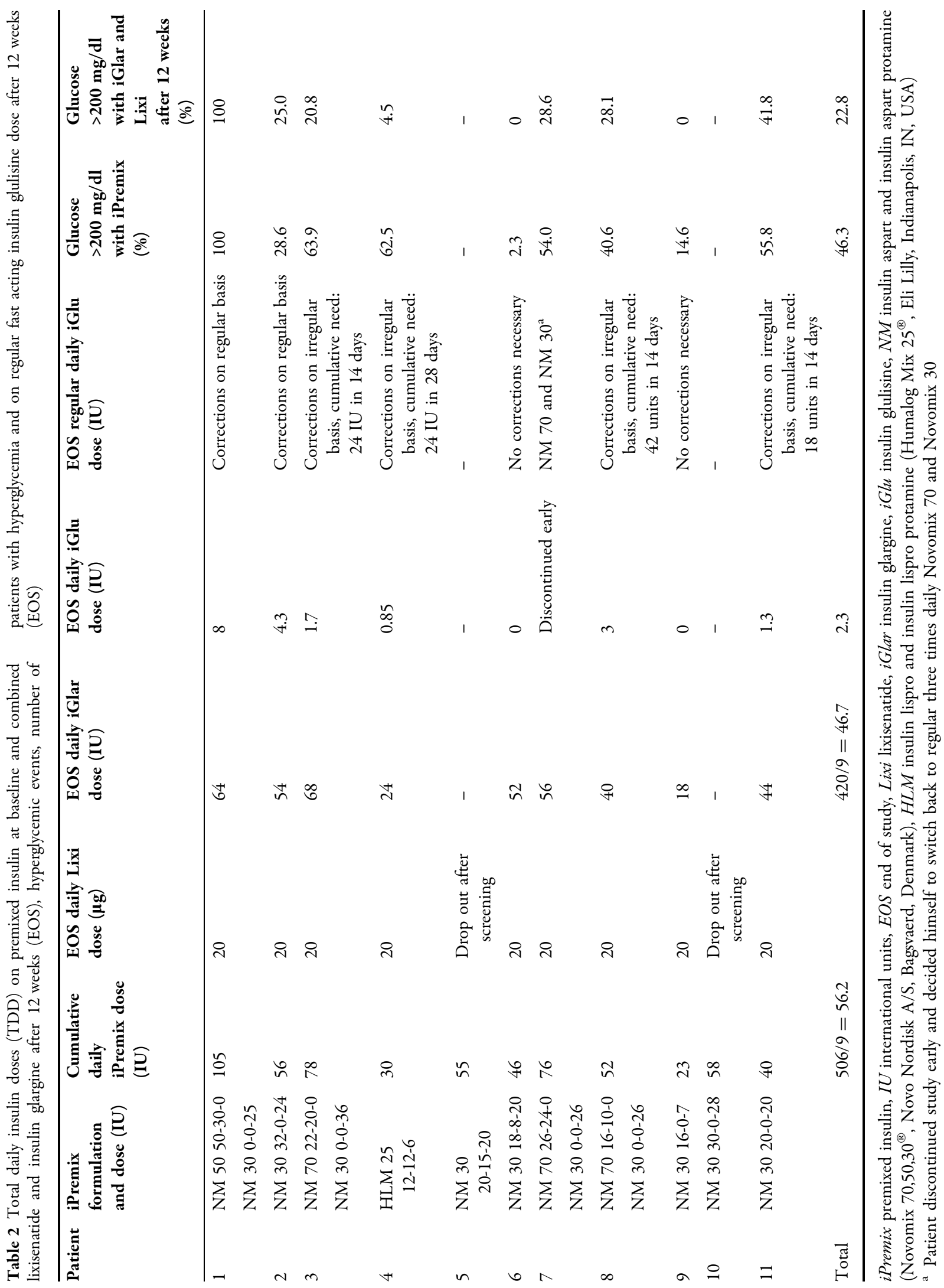


and lower C-peptide concentrations, which proposes a limitation of beta cell secretary function $[13,14]$. However, reports of associations of duration of T2DM with GLP1-RA response are heterogeneous, as low and high diabetes duration as well as no association were reported [15]. We are not able to show C-peptide levels; however, we observed higher response defined by A1c reduction of at least $0.4 \% \quad$ (19 $\mathrm{mmol} / \mathrm{mol}$; i.e., responders $)$ in patients with higher baseline A1c level (responders vs non responders: $9.6 \%$ vs $8.3 \%, 81$ vs $67 \mathrm{mmol} / \mathrm{mol} p<0.05, \quad U$ test), lower but insignificant baseline BMI (30.2 vs $31.9 \mathrm{~kg} / \mathrm{m}^{2}$, ns), comparable diabetes duration (12.6 vs 11.5 years, ns) and baseline insulin dose ( 54 vs $58 \mathrm{IU}, \mathrm{ns})$.

However, because of the pilot and proof of principle character of this study a few limitations are evident. First of all we did not conduct an RCT. As we intended to prove the feasibility of the conversion of the glucose-lowering therapy in an outpatient setting we decided to investigate this in an observational study design. Thus no direct comparison of effects is possible as no direct control group is available and our assumptions are based on these observations. Moreover, as a result of the study design we have a low intraindividual variance using dependent variables with comparison of pre and post combined therapy. Furthermore the durations of home glucose profiling receiving either iPremix (1 week) or combined treatment (12 weeks) were different, making direct comparisons difficult. Finally, the reliability of self-monitoring of blood glucose (SMBG) is limited.

The basal insulin dose was initially set at $60 \%$ of the daily iPremix. As shown in Fig. 1 we constantly had to increase the iGlar dose throughout the study to decrease high fasting glucose levels. Because of insufficient iGlar doses the majority of patients had to correct with fast acting iGlu at least on an irregular daily basis. This aspect might also be a reasonable explanation for the unsatisfactory fasting glucose levels after 12 weeks on combined Lixi and iGlar in our study collective, whereas recently published LixiLan trials could demonstrated sufficient reduction of fasting glucose
$[7,8]$. As a consequence no reduction of insulin dose seems to be necessary and should be performed in future investigations. Another weakness of the study is the very low number of patients included, which makes generalization of results difficult. However, this study was only designed to prove the principle of safe treatment potential of combined GLP1-RA and long-acting basal insulin analogue, which was demonstrated with our results.

\section{CONCLUSION}

Our study demonstrates safe application of combined Lixi and iGlar in patients with poorly controlled T2DM treated with at least twicedaily iPremix. Our patients had comparable glycemic control at the end of the treatment period, with ameliorated postprandial glucose profiles throughout the day, which resolves in the afternoon. Larger and sufficiently powered RCTs are needed to confirm these findings.

\section{ACKNOWLEDGEMENTS}

This study was sponsored by the Medical University of Vienna, Department of Internal Medicine III, Division of Endocrinology and Metabolism. The study was in part supported by Sanofi-aventis, Austria, SATURN Tower, Leonard-Bernstein-Straße 10, 1220 Vienna, Austria (study medication). These funders had no role in any aspect of the study beyond funding. All authors had full access to all of the data in this study and take complete responsibility for the integrity of the data and accuracy of the data analysis. There was no funding for article processing charges.

All named authors meet the International Committee of Medical Journal Editors (ICMJE) criteria for authorship for this manuscript, take responsibility for the integrity of the work as a whole, and have given final approval to the version to be published.

The authors thank the nurses, patients, and health professionals collaborating in the recruitment and support for this study. 
Disclosures. Anton Luger has received honoraries for lectures and/or consulting from Sanofi-aventis, Novo Nordisk, and Eli Lilly. Michael Rupert Krebs has received research support from Sanofi, AstraZeneca, and Ionis as well as speaker and consulting fees from AstraZeneca, Novartis, Novo Nordisk, Lilly, and Sanofi.

Peter Wolf, Lana Kosi-Trebotic, Albert Lukas, Yvonne Winhofer, Alexandra Kautzky-Willer, and Jürgen Harreiter have no conflicts of interest.

Compliance with Ethics Guidelines. All procedures followed were in accordance with the ethical standards of the responsible committee on human experimentation (Research Ethics Committee of the Medical University Vienna and Austrian Federal Office for Safety in Health Care) and with the Helsinki Declaration of 1964, as revised in 2013. Informed consent was obtained from all patients for being included in the study.

Data Availability. The datasets during and/ or analyzed during the current study are available from the corresponding author on reasonable request.

Open Access. This article is distributed under the terms of the Creative Commons Attribution-NonCommercial 4.0 International License (http://creativecommons.org/licenses/ by-nc/4.0/), which permits any noncommercial use, distribution, and reproduction in any medium, provided you give appropriate credit to the original author(s) and the source, provide a link to the Creative Commons license, and indicate if changes were made.

\section{REFERENCES}

1. International Diabetes Federation. IDF diabetes atlas 7th edition. 2016. http://www.diabetesatlas. org/. Accessed 2 Sept 2016.

2. Inzucchi SE, Bergenstal RM, Buse JB, et al. Management of hyperglycaemia in type 2 diabetes: a patient-centered approach. Position statement of the American Diabetes Association (ADA) and the European Association for the Study of Diabetes (EASD). Diabetologia. 2012;55(6):1577-96.

3. American Diabetes Association. Strategies for improving care. Diabetes Care. 2016;39(Suppl 1):S6-12.

4. Bellido V, Suarez L, Rodriguez MG, et al. Comparison of basal-bolus and premixed insulin regimens in hospitalized patients with type 2 diabetes. Diabetes Care. 2015;38(12):2211-6.

5. Riddle MC, Forst $\mathrm{T}$, Aronson R, et al. Adding once-daily lixisenatide for type 2 diabetes inadequately controlled with newly initiated and continuously titrated basal insulin glargine: a 24-week, randomized, placebo-controlled study (GetGoal-Duo 1). Diabetes Care. 2013;36(9):2497-503.

6. Kalra S, Gupta Y. Injectable coformulations in diabetology. Diabetes Ther. 2015;6(2):101-11.

7. Rosenstock J, Diamant M, Aroda VR, et al. Efficacy and safety of LixiLan, a titratable fixed-ratio combination of lixisenatide and insulin glargine, versus insulin glargine in type 2 diabetes inadequately controlled on metformin monotherapy: the LixiLan proof-of-concept randomized trial. Diabetes Care. 2016;39(9):1579-86.

8. Rosenstock J, Aronson R, Grunberger G, et al. Benefits of LixiLan, a titratable fixed-ratio combination of insulin glargine plus lixisenatide, versus insulin glargine and lixisenatide monocomponents in type 2 diabetes inadequately controlled on oral agents: the LixiLan-O randomized trial. Diabetes Care. 2016;39(11):2026-35.

9. Aroda VR, Rosenstock J, Wysham C, et al. Efficacy and safety of LixiLan, a titratable fixed-ratio combination of insulin glargine plus lixisenatide in type 2 diabetes inadequately controlled on basal insulin and metformin: the LixiLan-L randomized trial. Diabetes Care. 2016;39(11):1972-80.

10. Riddle $\mathrm{MC}$, Aronson R, Home $\mathrm{P}$, et al. Adding once-daily lixisenatide for type 2 diabetes inadequately controlled by established basal insulin: a 24-week, randomized, placebo-controlled comparison (GetGoal-L). Diabetes Care. 2013;36(9):2489-96.

11. American Diabetes Association, Standards of medical care in diabetes-2017. Diabetes Care. 2017;40(Suppl 1):S64-74.

12. Ahren B, Leguizamo Dimas A, Miossec P, Saubadu S, Aronson R. Efficacy and safety of lixisenatide once-daily morning or evening injections in type 2 
diabetes inadequately controlled on metformin (GetGoal-M). Diabetes Care. 2013;36(9):2543-50.

13. Davis SN, Johns D, Maggs D, et al. Exploring the substitution of exenatide for insulin in patients with type 2 diabetes treated with insulin in combination with oral antidiabetes agents. Diabetes Care. 2007;30(11):2767-72.

14. Drucker DJ, Nauck MA. The incretin system: glucagon-like peptide-1 receptor agonists and dipeptidyl peptidase- 4 inhibitors in type 2 diabetes. The Lancet. 2006;368(9548):1696-705.

15. Jones AG, Shields BM, Hyde CJ, Henley WE, Hattersley AT. Identifying good responders to glucose lowering therapy in type 2 diabetes: implications for stratified medicine. PLoS One. 2014;9(10): e111235. 